IFN Working Paper No. 1274, 2019

\title{
Samuelson's Approach to Revealed Preference Theory: Some Recent Advances
}

Thomas Demuynck and Per Hjertstrand 


\title{
Samuelson's Approach to Revealed Preference Theory: Some Recent Advances
}

\author{
Thomas Demuynck* $\quad$ Per Hjertstrand ${ }^{\dagger}$
}

April, 2019

Forthcoming chapter in Paul Samuelson: Master of Modern Economics,
R.G. Anderson, W.A. Barnett and R.A. Cord (Eds.), Palgrave
Macmillan, London

\begin{abstract}
Since Paul Samuelson introduced the theory of revealed preference, it has become one of the most important concepts in economics. This chapter surveys some recent contributions in the revealed preference literature. We depart from Afriat's theorem, which provides the conditions for a data set to be consistent with the utility maximization hypothesis. We provide and motivate a new condition, which we call the Varian inequalities. The advantage of the Varian inequalities is that they can be formulated as a set of mixed integer linear inequalities, which are linear in the quantity and price data. We show how the Varian inequalities can be used to derive revealed preference tests for weak separability, and show how it can be used to formulate tests of the collective household model. Finally, we discuss measurement errors in the observed data and measures of goodness-of-fit, power and predictive success.
\end{abstract}

Keywords: Afriat's theorem; Collective household model; GARP; Mixed integer linear programing; Revealed preference; Varian inequalities; Weak separability

\section{Introduction}

In January 2005, Hal Varian (Varian 2006) searched the JSTOR business and economics journals and Google Scholar for the phrase 'revealed preference'. He reports to have found 997 articles in JSTOR and approximately 3,600 works on Google Scholar containing this

*ECARES, Université Libre de Bruxelles. Avenue F.D. Roosevelt, CP 114, B-1050 Brussels, Belgium. E-mail: thomas.demuynck@ulb.ac.be. Thomas Demuynck acknowledges financial support by the Fonds de la Recherche Scientifique-FNRS under grant nr F.4516.1.

${ }^{\dagger}$ Research Institute of Industrial Economics (IFN). P.O. Box 55665, SE-102 15 Stockholm, Sweden. E-mail: Per.Hjertstrand@ifn.se. Per Hjertstrand acknowledges financial support from Jan Wallander och Tom Hedelius stiftelse, Marcus och Marianne Wallenbergs stiftelse and Johan och Jakob Söderbergs stiftelse. 
phrase. Based on this result, he concluded that "Surely, revealed preference must count as one of the most influential ideas in economics" (ibid.: 99; italics added). A March 2018 search of the same phrase over the period from January 2005 to March 2018 found an additional 996 articles in JSTOR business and economics journals and an additional 22,200 works on Google Scholar. ${ }^{1}$ Echoing Varian's view, we can confidently state that revealed preference continues to be an important and influential concept in economics.

Since its introduction by Paul Samuelson in 1938, revealed preference has been applied to a vast number of different areas. Varian (ibid.) surveys the early history of the literature that evolved from Samuelson's initial contribution. The aim of this chapter is to continue where Varian left off and survey some of the more recent advances in the theoretical and applied revealed preference literature. ${ }^{2}$

\section{$2 \quad$ Testing for Rationality}

Revealed preference theory, initiated by Samuelson $(1938,1948)$, provides a structural approach to analyze demand behavior. Its main underlying principle is that a consumer's observed choices provide information about her underlying preferences. If a consumer is observed to have chosen a certain consumption bundle $x{ }^{3}$ while another bundle $y$ was also available (e.g. because it was less expensive), then she reveals her preference for $x$ over $y$. Equivalently, we say that $x$ is revealed preferred over $y$. In this manner, choices say something about the underlying preferences of the consumer. Samuelson (1938) introduced the weak axiom of revealed preference (WARP), which provides a test of the simplest form of the utility maximization hypothesis: if a bundle $x$ is revealed preferred over a bundle $y$, then at some other instance, $y$ should not be revealed preferred over $x$. WARP requires the revealed preference relation to be asymmetric. Houthakker (1950) generalized WARP by introducing the strong axiom of revealed preference (SARP) which states that the revealed preference relation is acyclic. Interestingly, he also showed that this gives the strongest test for the consistency of choice behavior under the utility maximization hypothesis.

\subsection{Afriat's Theorem}

Samuelson and Houthakker derived their results under the assumption that one can observe the entire demand function of a consumer. In a subsequent seminal contribution to the literature, Afriat (1967) considered the more realistic setting where the researcher only observes a finite set of choices. Starting from such a finite data set, Afriat showed that a slight relaxation of SARP, which he calls cyclical consistency, provides the necessary and sufficient conditions for the existence of a utility function whose imposed choice behavior is consistent with the data. Diewert (1973) and Varian (1982) made Afriat's

\footnotetext{
1 The more refined search 'revealed preference' + samuelson returned approximately 4,460 works on Google Scholar over the period from 2005 to 2018.

2 Other recent surveys or overviews of revealed preference, some with a different focus, are Cherchye et al. (2009a), Diewert (2012), Crawford and De Rock (2014) and Chambers and Echenique (2016).

${ }^{3}$ By a bundle we mean an $N$-dimensional vector that gives the various quantities over the $N$ goods that the consumer chooses.
} 
approach more transparent and gave complete proofs. It was also Varian (ibid.) who used the term general axiom of revealed preference (GARP) instead of cyclical consistency.

Before we present a more formal exposition of Afriat's results, we need to introduce the concept of a revealed preference relation.

Definition 1 (Revealed Preference) Given a finite data set $S=\left(p^{t}, x^{t}\right)_{t=1, \ldots, T}$ of prices $p^{t} \in \mathbb{R}_{++}^{N}$ and consumption bundles $x^{t} \in \mathbb{R}_{+}^{N}$, we say that $x^{t}$ is directly revealed preferred to a bundle $x$ (written $x^{t} R_{D} x$ ) if $p^{t} x^{t} \geq p^{t} x{ }^{4}$ We say that $x^{t}$ is revealed preferred to $x$ (written $x^{t} R x$ ) if there is some (possibly empty) sequence $u, v, \ldots, s$ such that $p^{t} x^{t} \geq p^{t} x^{u}, p^{u} x^{u} \geq p^{u} x^{v}, \ldots, p^{s} x^{s} \geq p^{s} x$. The revealed preference relation $R$ is the transitive closure of the direct revealed preference relation $R_{D}$.

The intuition behind the revealed preference relation is simple. If $p^{t} x^{t} \geq p^{t} x$, then $x^{t}$ was chosen (at observation $t$ ) while the bundle $x$ was not more expensive to buy. Given that the consumer chose the bundle $x^{t}$ and not $x$, it must have been her preferred option.

Theorem 1 (Afriat's Theorem) Given a finite data set of observed prices and choices $S=\left(p^{t}, x^{t}\right)_{t=1, \ldots, T}$, the following conditions are equivalent:

1. There exists a locally non-satiated ${ }^{5}$ utility function $u(x)$ that rationalizes the data set $S$, i.e. for all observations $t$ and all bundles $x$, if $p^{t} x^{t} \geq p^{t} x$ then, $u\left(x^{t}\right) \geq u(x)$.

2. The data set $S$ satisfies the generalized axiom of revealed preference (GARP), i.e. for all observations $t$ and $s$, if $x^{t} R x^{s}$ then, $p^{s} x^{s} \leq p^{s} x^{t}$.

3. For all observations $t$, there exists a number $U^{t}$ and a number $\lambda^{t}>0$ such that the Afriat inequalities hold, i.e. for all observations $t$ and $s$,

$$
U^{s}-U^{t} \leq \lambda^{t} p^{t}\left(x^{s}-x^{t}\right)
$$

4. For all observations $t$, there exists a number $V^{t}$ such that the Varian inequalities hold, i.e. for all observations $s$ and $t$,

$$
\begin{aligned}
& \text { if } p^{t} x^{t} \geq p^{t} x^{s} \text { then, } V^{t} \geq V^{s}, \\
& \text { if } p^{t} x^{t}>p^{t} x^{s} \text { then, } V^{t}>V^{s} .
\end{aligned}
$$

5. There exists a continuous, monotone and concave utility function $u(x)$ that rationalizes the data.

Conditions 1, 2, 3 and 5 give the standard version of Afriat's theorem. The equivalence between conditions 1 and 5 shows that continuity, monotonicity and concavity are nontestable properties in settings with linear budgets. ${ }^{6}$

${ }^{4}$ By $p x$, we mean the dot product $\sum_{i=1}^{N} p_{i} x_{i}$.

${ }^{5}$ A function $u: \mathbb{R}_{+}^{N} \rightarrow \mathbb{R}$ is locally non-satiated if for all $x \in \mathbb{R}_{+}^{N}$ there exists a neighborhood $\mathcal{N}_{x}$ around $x$ and a vector $y \in \mathcal{N}_{x} \cap \mathbb{R}_{+}^{N}$ such that $u(y)>u(x)$.

${ }^{6}$ With non-linear budgets, the property of quasi-concavity is testable (see Cherchye et al. 2014). 
Afriat's theorem suggests various procedures to test the utility maximization model. First, the GARP in condition 2 offers a simple combinatorial test on observed prices and quantities. Varian (1982) provides a simple and fast algorithm to implement GARP based on Warshall (1962)'s algorithm to compute the transitive closure of a relation. ${ }^{7}$

The Afriat inequalities in condition 3 gives an alternative equivalent condition that takes the form of a set of linear inequalities. The variables $U^{t}$ and $\lambda^{t}$ can be interpreted as the utility and marginal utility of income values at the observation $t$. To see this, suppose that the data $S=\left(p^{t}, x^{t}\right)_{t=1, \ldots, T}$ were generated by a differentiable and concave utility function $u(x)$, in which case, by the properties of concavity, it must be that for all observations $t, s$ :

$$
u\left(x^{s}\right)-u\left(x^{t}\right) \leq \nabla u\left(x^{t}\right)\left(x^{s}-x^{t}\right) .
$$

Additionally, the first-order conditions for utility maximization subject to a linear budget constraint (excluding boundary conditions) yield:

$$
\nabla u\left(x^{t}\right)=\lambda^{t} p^{t},
$$

where $\lambda^{t}$ is the marginal utility of income (i.e. the Lagrange multiplier). If we substitute this into the concavity inequalities and define $U^{t}=u\left(x^{t}\right)$, we effectively obtain the Afriat inequalities. Diewert (1973) and Fleissig and Whitney (2005) propose linear programing (LP) procedures to check whether there exists a feasible solution to the Afriat inequalities.

Condition 4 is usually omitted from Afriat's theorem, but it turns out to be a key formulation for various more recent developments in revealed preference theory. It was originally introduced in the non-parametric production literature by Varian (1984), which motivates the use of the term 'Varian inequalities'.

The intuition behind the Varian inequalities is straightforward if one interprets $V^{t}$ as the utility of bundle $x^{t}$. Then, if $p^{t} x^{t} \geq p^{t} x^{s}$, we have that $x^{t}$ is directly revealed preferred to $x^{s}$, so $V^{t}=u\left(x^{t}\right)$ should be at least as large as the utility $V^{s}=u\left(x^{s}\right)$. If $p^{t} x^{t}>p^{t} x^{s}$, then a similar reasoning (together with local non-satiation) guarantees that $V^{t}>V^{s}$.

A second intuition for why $V^{t}$ can be interpreted as the utility value at observation $t$ follows a similar reasoning as for the Afriat inequalities. Suppose that the data $S=$ $\left(p^{t}, x^{t}\right)_{t=1, \ldots, T}$ were generated by a nonsatiated, differentiable and quasiconcave utility function $u(x) .{ }^{8}$ Differentiability and quasiconcavity imply:

$$
\text { if } u\left(x^{s}\right)-u\left(x^{t}\right) \geq 0 \text {, then } \nabla u\left(x^{t}\right)\left(x^{s}-x^{t}\right) \geq 0 \text {, }
$$

while nonsatiation further implies:

$$
\text { if } u\left(x^{s}\right)-u\left(x^{t}\right)>0 \text {, then } \nabla u\left(x^{t}\right)\left(x^{s}-x^{t}\right)>0 \text {. }
$$

Substituting the first-order conditions for utility maximization and defining $V^{t}$ for all observations $t$ as utility values, i.e. $V^{t}=u\left(x^{t}\right)$, yields:

$$
\begin{aligned}
& \text { if } V^{s}-V^{t} \geq 0, \text { then } \lambda^{t} p^{t}\left(x^{s}-x^{t}\right) \geq 0, \\
& \text { if } V^{s}-V^{t}>0 \text {, then } \lambda^{t} p^{t}\left(x^{s}-x^{t}\right)>0 .
\end{aligned}
$$

7 Varian's algorithm is widely available in many programming languages. See, for example, www.revealedpreferences.org for codes.

${ }^{8}$ A nonsatiated and quasiconcave function is also called semistrict quasiconcave (see Ginsberg 1973). 
However, since $\lambda^{t}>0$, we can without loss of generality drop it from the inequalities. Taking the contrapositive, the Varian inequalities follow.

Analogous to the Afriat inequalities, it is possible to check whether the Varian inequalities have a feasible solution by solving an LP problem. ${ }^{9}$ Interestingly, this LP problem only has $T$ parameters, which is $T$ fewer than the LP procedure to test the Afriat inequalities.

Cherchye et al. (2015a) show that the Varian inequalities can be equivalently formulated in terms of the following feasibility problem:

There exist numbers $V^{t} \in[0,1]$ and $X^{t, s} \in\{0,1\}$ such that for all observations $t, s$ :

$$
\begin{aligned}
& V^{t}-V^{s}<X^{t, s}, \\
& \left(X^{t, s}-1\right) \leq V^{t}-V^{s}, \\
& p^{t}\left(x^{t}-x^{s}\right)<X^{t, s} A^{t}, \\
& \left(X^{t, s}-1\right) A^{s} \leq p^{s}\left(x^{t}-x^{s}\right) .
\end{aligned}
$$

Here $A^{t}$ is a fixed number greater than $p^{t} x^{t}$. The variables $X^{t, s}$ are either 0 or 1 . The interpretation is that $X^{t, s}$ should be equal to one if and only if $V^{t} \geq V^{s}$. This condition is guaranteed by the first and second inequalities: the first inequality ensures that $V^{t} \geq V^{s}$ implies $X^{t, s}=1$, while the second condition inequality that $X^{t, s}=1$ implies $V^{t} \geq V^{s}$. The third inequality guarantees that if $p^{t} x^{t} \geq p^{t} x^{s}$, then $X^{t, s}=1$ (i.e. $V^{t} \geq V^{s}$ ). This constitutes the first Varian inequality (i.e. condition (1)). The last inequality guarantees that $X^{t, s}=1$ (i.e. $V^{t} \geq V^{s}$ ) implies $p^{s} x^{t} \geq p^{s} x^{s}$, which is the contrapositive of the second Varian inequality (i.e. condition (2)).

The above feasibility problem cannot be verified using LP methods because the variables $X^{t, s}$ are restricted to be either 0 or 1 . On the other hand, they can be verified using mixed integer linear programing algorithms (MILP). Similar to a standard LP problem, an MILP problem minimizes a linear objective function subject to a set of linear constraints. However, in contrast to an LP problem, the variables in an MILP problem may also take integer values. From a computational perspective, MILP algorithms are much less efficient than LP algorithms. ${ }^{10}$ Even so, this MILP formulation has been shown to be very useful in practice. Its main advantage is that the system of inequalities remains linear even if some prices or quantities become unobserved (i.e. are treated as parameters in the problem). This is the case for many revealed preference characterizations of more complicated behavioral models as we will illustrate below.

\section{$3 \quad$ Extensions and Generalizations}

A sizable part of the revealed preference literature has focused on enlarging the class of economic models to which the revealed preference methodology can be applied. As

\footnotetext{
${ }^{9}$ Without loss of generality, the strict inequality on the right-hand side in the second row can be converted to a weak inequality by noticing that the inequalities are homogeneous of degree one in $V^{t}$. Thus, the second row may be written as if $p^{t}\left(x^{t}-x^{s}\right)>0$, then $V^{t}-V^{s} \geq \varepsilon$, for any $\varepsilon>0$ (the standard choice is to set $\varepsilon=1$ ).

${ }^{10}$ From a computational perspective, we have that LP problems are solvable in polynomial time, while MILP problems are in general NP-hard.
} 
the present space is too limited to discuss all of them, we will focus in this section on two of these models: the weakly separable utility maximization model and the collective household model. At the end of the section, we provide a brief, non-exhaustive overview of other recent contributions to the literature.

\subsection{Revealed Preference Tests for Weak Separability}

Weak separability is a key property in economics. Every empirical study involving consumption, macro or financial data is based, either explicitly or implicitly, on the assumption that preferences are at least weakly separable. A standard example is when non-durable goods are analyzed without reference to durable goods or when consumption is analyzed without reference to labor supply. In such settings, the former set of goods is assumed to be weakly separable from the latter. We say that a group of goods is weakly separable from all other goods when the marginal rate of substitution between any two goods in the separable group does not depend on the quantities consumed of the goods outside the group. Hence, weak separability implies that the demand functions for the goods in the separable group depend only on the prices of those goods and total expenditure allocated to this group.

Revealed preference methods are a particularly suitable means of testing for weak separability and other forms of separability. The revealed preference conditions for separability were derived by Afriat (1969), Varian (1983a) and Diewert and Parkan (1985). Suppose that we partition the quantities $x$ into two mutually exclusive and collectively exhaustive groups, given by $y$ and $z$. Denote the corresponding prices of $y$ by $q$ and those of $z$ by $r$, respectively. We say that the utility function $u(y, z)$ is weakly separable in the $y$-goods if there exists a macro (aggregator) function $v$ and a sub-utility function $w$ such that

$$
u(y, z)=v(w(y), z) .
$$

We have the following revealed preference characterization.

Theorem 2 For any finite data set $S=\left(\left(q^{t}, r^{t}\right),\left(y^{t}, z^{t}\right)\right)_{t=1, \ldots, T}$, the following conditions are equivalent:

1. There exists a continuous, monotone, concave and weakly separable utility function $v(w(y), z)$ that rationalizes the data, i.e. for all observations $t$ and all $(y, z)$, if $q^{t} y^{t}+r^{t} z^{t} \geq q^{t} y+r^{t} z$, then $v\left(w\left(y^{t}\right), z^{t}\right) \geq v(w(y), z)$.

2. For all observations there exist numbers $V^{t}$ and $W^{t}$ and numbers $\mu^{t}>0$ and $\lambda^{t}>0$ such that for all observations $t$ and $s$,

$$
\begin{aligned}
& W^{s}-W^{t} \leq \mu^{t} q^{t}\left(y^{s}-y^{t}\right), \\
& V^{s}-V^{t} \leq \lambda^{t} r^{t}\left(z^{s}-z^{t}\right)+\frac{\lambda^{t}}{\mu^{t}}\left(W^{s}-W^{t}\right) .
\end{aligned}
$$

This theorem is analogous to Afriat's theorem. In fact, it is easy to see that condition

2 mirrors condition 3 in Theorem 1 . To grasp the intuition behind condition 2, consider 
the two concavity restrictions:

$$
\begin{aligned}
& v\left(w\left(y^{s}\right), z^{s}\right)-v\left(w\left(y^{t}\right), z^{t}\right) \leq \frac{\partial v\left(w\left(y^{t}\right), z^{t}\right)}{\partial w}\left(w\left(y^{s}\right)-w\left(y^{t}\right)\right)+\nabla_{z} v\left(w\left(y^{t}\right), z^{t}\right)\left(z^{s}-z^{t}\right), \\
& w\left(y^{s}\right)-w\left(y^{t}\right) \leq \nabla_{y} w\left(y^{t}\right)\left(y^{s}-y^{t}\right),
\end{aligned}
$$

and the (interior) first-order conditions

$$
\begin{aligned}
& \frac{\partial v\left(w\left(y^{t}\right), z^{t}\right)}{\partial w} \nabla_{y} w\left(y^{t}\right)=\lambda^{t} q^{t} \\
& \nabla_{z} v\left(w\left(y^{t}\right), z^{t}\right)=\lambda^{t} r^{t} \\
& \nabla_{y} w\left(y^{t}\right)=\mu^{t} q^{t} .
\end{aligned}
$$

From the first and third first-order conditions, we have $\frac{\partial v\left(w\left(y^{t}\right), z^{t}\right)}{\partial w} \mu^{t} q^{t}=\lambda^{t} q^{t}$, which implies that for any good in the separable $y$-group:

$$
\frac{\partial v\left(w\left(y^{t}\right), z^{t}\right)}{\partial w}=\frac{\lambda^{t}}{\mu^{t}}
$$

Substituting this and the other first-order conditions into the concavity inequalities, and setting $v\left(w\left(y^{s}\right), z^{s}\right)=V^{s}, v\left(w\left(y^{t}\right), z^{t}\right)=V^{t}, w\left(y^{t}\right)=W^{t}$ and $w\left(y^{s}\right)=W^{s}$ gives the inequalities (3) and (4).

Although condition 2 provides a clear set of inequalities that need to be verified, doing so in practice is far from trivial. The problem concerns inequality (4), which is no longer linear because it contains the term $\lambda^{t} / \mu^{t}$. Nevertheless, Swofford and Whitney (1994) proposed to jointly check whether there exists a solution to inequalities (3) and (4). Their procedure is further extended to allow for incomplete adjustment of expenditure and therefore to account for various forms of habit persistence such as adjustment costs and the formation of expectations. However, because it is based on finding a solution to the non-linear inequalities (3) and (4), any implementation is based on solving a complex non-linear optimization problem, which may be computationally difficult even for medium-sized data sets. ${ }^{11}$

An alternative sequential approach was proposed by Varian (1983a). This two-step method is based on the fact that $W^{t}$ and $1 / \mu^{t}$ can be interpreted as quantity and price indices for the separable $y$-group of goods. The first step consists of finding numbers $W^{t}$ and $\mu^{t}$ that satisfy the Afriat inequalities (3), and the second step consists of using these numbers as plug-ins to check whether inequality (4) holds. Since $W^{t}$ and $\mu^{t}$ are known variables in the second step, verifying whether (4) holds is a linear problem and, by Theorem 1 , is equivalent to testing whether the data set

$$
S^{\prime}=\left(\left(r^{t}, 1 / \mu^{t}\right),\left(z^{t}, W^{t}\right)\right)_{t=1, \ldots, T} \text { satisfies } G A R P .
$$

Varian (1983a) suggested a combinatorial algorithm to calculate the numbers $W^{t}$ and $\mu^{t}$ in the first step while Fleissig and Whitney (2003) proposed a linear programing procedure to find these numbers.

11 Cherchye et al. (2015a) showed that verifying conditions (3) and (4) is an NP-hard problem, meaning that it is computationally intractable for very large datasets. See also Echenique (2014). 
Unfortunately, this sequential approach is biased towards rejecting weak separability (see, for example, Hjertstrand 2009). The bias arises because the numbers $W^{t}$ and $\mu^{t}$ in the first step are constructed without reference to the GARP test in the second step. Specifically, the degree of the bias is related to the choice of $W^{t}$ and $\mu^{t}$, and since these numbers are not unique, some choices may cause a violation of weak separability while others may not. Using Monte Carlo experiments, Hjertstrand (2009) found that Varian (1983a)'s procedure is heavily biased while Fleissig and Whitney (2003)'s procedure is considerably less so.

However, all is not lost. As shown in Cherchye et al. (2015a), we can use the MILP formulation of the Varian inequalities in Theorem 1 to derive an exact test of conditions (3) and (4). To see this, first note that the data set $S^{\prime}$ satisfies GARP even if all prices $\left(r^{t}, 1 / \mu^{t}\right)$ are multiplied by a common number (i.e. the GARP test is homogeneous of degree zero in prices). Thus, the data set $S^{\prime}$ satisfies GARP if and only if

$$
S^{\prime \prime}=\left(\left(\mu^{t} r^{t}, 1\right),\left(z^{t}, W^{t}\right)\right)_{t=1, \ldots, T} \text { satisfies GARP. }
$$

By the equivalence of GARP and the Varian inequalities in Theorem 1, it is then easy to see that the Varian inequalities are linear even if we do not observe the 'prices' $\mu^{t} r^{t}$ or the 'quantities' $W^{t}$. Thus, the MILP formulation of the Varian inequalities gives the following practical test of the weak separability conditions in Theorem 2:

There exist numbers $0 \leq V^{t} \leq 1,0 \leq W^{t} \leq 1$ and $0<\mu^{t} \leq 1$ and binary numbers $X^{t, s} \in\{0,1\}$ such that the following inequalities hold for all observations $s$ and $t$,

$$
\begin{aligned}
& W^{s}-W^{t} \leq \mu^{t} q^{t}\left(y^{s}-y^{t}\right), \\
& V^{t}-V^{s}<X^{t, s}, \\
& \left(X^{t, s}-1\right) \leq V^{t}-V^{s}, \\
& \mu^{t} r^{t}\left(z^{t}-z^{s}\right)+\left(W^{t}-W^{s}\right)<X^{t, s} A^{t}, \\
& \left(X^{t, s}-1\right) A^{s} \leq \mu^{s} r^{s}\left(z^{t}-z^{s}\right)+\left(W^{t}-W^{s}\right),
\end{aligned}
$$

where $A^{t}>r^{t} z^{t}+1$. The first inequality equals the Afriat inequalities (3) in Theorem 2, while the last four inequalities ensure that the data set $S^{\prime \prime}$ satisfies GARP.

The revealed preference conditions of Theorem 2 have been applied in different areas. Swofford and Whitney (1987), Belongia and Chrystal (1991) and Fisher and Fleissig (1997) apply Varian (1983a)'s procedure, while Jones et al. (2005) and Elger et al. (2008) apply the procedures developed by Fleissig and Whitney (2003) and Swofford and Whitney (1994) to test whether various monetary aggregates are weakly separable to analyze what monetary assets the public is including in their definition of 'money'.

More recently, Hjertstrand et al. (2016) apply the MILP procedure to identify monetary aggregates. Using the same data, Hjertstrand et al. (2019) modify the MILP procedure to check how close monetary and consumption aggregates calculated from superlative and other index numbers are to being weakly separable. These recent papers show that Cherchye et al. (2015a)'s MILP procedure can be effectively implemented on medium- and large-scale data sets.

Hjertstrand and Swofford (2012) derive revealed preference conditions for weak separability of the indirect utility function. In Cherchye et al. (2015a), it is also shown how 
the MILP procedure can be modified to test for weak separability in the indirect utility function and how the procedure can be adapted when the sub-utility function $w(y)$ is assumed to be homothetic. Hjertstrand and Swofford (2019) apply these test procedures to check whether some monetary and consumption aggregates are indirectly and homothetically weakly separable.

\subsection{Collective Household Models}

The traditional literature on household consumption behavior assumes that households behave as single agents. This unitary model of household consumption imposes empirically testable restrictions on consumption behavior (e.g. Slutsky symmetry or GARP) that are frequently rejected empirically. Because of this, the literature has shifted to models that explicitly take into account the fact that households are composed of distinct individuals and that each member has his or her own preferences. Household decisions are then determined by an underlying intra-household bargaining process. Following Apps and Rees (1988) and Chiappori $(1988,1992)$ the intra-household process is usually assumed to produce a Pareto efficient allocation of resources in the household.

Consider a household with two members, $A$ and $B$, with utility functions $u_{A}$ and $u_{B}$ over own consumption. The benchmark model assumes that goods can be partitioned into private goods and (household-level) public goods. Private goods are exclusive, meaning that a good can only be consumed by one household member (e.g. food, clothing). Public goods are non-exclusive and are jointly consumed by both members (e.g. housing, heating, cleaning). Denote private goods by $q$ and public goods by $Q$ with prices $p$ and $P$. Assuming Pareto-efficient household decision making, the household chooses the allocation $\left(q_{A}, q_{B}, Q\right)$ that maximizes a weighted sum of utilities.

$$
\max _{q^{A}, q^{B}, Q} u_{A}\left(q_{A}, Q\right)+\mu u_{B}\left(q_{B}, Q\right) \text { s.t. } p\left(q_{A}+q_{B}\right)+P Q \leq Y,
$$

where $Y$ is the household income and $\mu$ is the Pareto weight that might depend on prices and income.

The characterization of this collective model was established in a series of papers by Cherchye et al. (2007, 2009b, 2011a).

Theorem 3 (Cherchye et al.) For any finite data set $S=\left(\left(p^{t}, P^{t}\right),\left(q^{t}, Q^{t}\right)\right)_{t=1, \ldots, T}$, the following conditions are equivalent:

1. There exist continuous, monotone and concave utility functions $u_{A}$ and $u_{B}$, Pareto weights $\mu^{t}$ and private consumption bundles $q_{A}^{t}$ and $q_{B}^{t}$ that rationalize the data, i.e. for all observations $t, q_{A}^{t}+q_{B}^{t}=q^{t}$, and for all $\left(q_{A}, q_{B}, Q\right)$ if $p^{t} q^{t}+P^{t} Q^{t} \geq$ $p^{t}\left(q_{A}+q_{B}\right)+P^{t} Q$, then

$$
u_{A}\left(q_{A}^{t}, Q^{t}\right)+\mu^{t} u_{B}\left(q_{B}^{t}, Q^{t}\right) \geq u_{A}\left(q_{A}, Q\right)+\mu^{t} u_{B}\left(q_{B}, Q\right) .
$$

2. For all observations $t$, there are numbers $U_{A}^{t}, U_{B}^{t}$ and $\lambda_{A}^{t}, \lambda_{B}^{t}>0$, consumption 
bundles $q_{A}^{t}, q_{B}^{t}$ and prices $P_{A}^{t}, P_{B}^{t}$ such that for all observations $t$ and $s$ :

$$
\begin{aligned}
& U_{A}^{s}-U_{A}^{t} \leq \lambda_{A}^{t} p^{t}\left(q_{A}^{s}-q_{A}^{t}\right)+\lambda_{A}^{t} P_{A}^{t}\left(Q^{s}-Q^{t}\right), \\
& U_{B}^{s}-U_{B}^{t} \leq \lambda_{B}^{t} p^{t}\left(q_{B}^{s}-q_{B}^{t}\right)+\lambda_{B}^{t} P_{B}^{t}\left(Q^{s}-Q^{t}\right), \\
& q_{A}^{t}+q_{B}^{t}=q^{t} \\
& P_{A}^{t}+P_{B}^{t}=P^{t}
\end{aligned}
$$

3. There exist private consumption bundles $q_{A}^{t}, q_{B}^{t}$ and public prices $P_{A}^{t}, P_{B}^{t}$ such that for all observations $t$,

$$
\begin{aligned}
& q_{A}^{t}+q_{B}^{t}=q^{t}, \\
& P_{A}^{t}+P_{B}^{t}=P^{t},
\end{aligned}
$$

and the data sets $S_{A}=\left(\left(p^{t}, P_{A}^{t}\right),\left(q_{A}^{t}, Q^{t}\right)\right)_{t=1, \ldots, T}$ and $S_{B}=\left(\left(p^{t}, P_{t}^{B}\right),\left(q_{B}^{t}, Q^{t}\right)\right)_{t=1, \ldots, T}$ satisfy GARP.

Condition 2 gives the usual Afriat inequalities. As before, they can be obtained by using concavity inequalities together with the first-order conditions for the household maximization problem. The numbers $P_{A}^{t}, P_{B}^{t}$ should be interpreted as the marginal willingness to pay (MWTP) at observation $t$ for public goods by members $A$ and $B$. Condition 3 uses Theorem 1 to translate the Afriat inequalities into equivalent GARP conditions. The intuition behind the revealed preference restrictions can be clarified by making use of the second fundamental theorem of welfare economics. This theorem states that any Pareto efficient allocation can be decentralized by making use of Lindahl prices for public goods, where the Lindahl prices sum to the market price. In our setting, these Lindahl prices are given by the MWTP vectors $P_{A}^{t}$ and $P_{B}^{t}$. Thus, by the second fundamental theorem the Pareto-optimal allocation is equivalent to a situation where each household member is maximizing his or her own utility utility function subject to the observed prices $p^{t}$ for private goods and the Lindahl prices $P_{A}^{t}\left(P_{B}^{t}\right)$ for public goods.

The GARP conditions in Theorem 3 are not directly verifiable because neither the Lindahl prices $P_{A}^{t}, P_{B}^{t}$ nor the private quantities $q_{A}^{t}, q_{B}^{t}$ are observed. However, the Varian inequalities corresponding to the GARP conditions in condition 3 do remain linear in prices and quantities, and therefore, the MILP procedure provides a feasible way to check the revealed preference conditions.

The model above assumes that household allocations are Pareto optimal. An alternative approach is to assume that household allocations are determined through a noncooperative Nash equilibrium where each member chooses her/his own amount of private goods $\left(q^{A}, q^{B}\right)$ and her/his own contribution to the level of public goods $\left(Q_{A}, Q_{B}\right)$. The total level of public goods in the household is then $Q_{A}+Q_{B}$. In this case, member $A$ solves

$$
\max _{q_{A}, Q_{A}} u_{A}\left(q_{A}, Q_{A}+Q_{B}\right) \text { s.t. } p q_{A}+P Q_{A} \leq\left(Y-p q_{B}-P Q_{B}\right) .
$$

A similar maximization problem holds for member $B$. As shown by Cherchye et al. (2011b), the revealed preference conditions for this non-cooperative model are very similar to those of the cooperative model except that in this case, the MWTP adding up condition,

$$
P_{A}^{t}+P_{B}^{t}=P^{t}
$$


is replaced by a condition where the maximum MWTP should equal the market price, i.e.

$$
\max \left\{P_{A}^{t}, P_{B}^{t}\right\}=P^{t}
$$

where the maximum operator is element-wise, and the numbers $P_{A}^{t}, P_{B}^{t}$ are the MWTP at observation $t$ for public goods by members $A$ and $B$. The non-cooperative model is analogous to the public goods model with voluntary contributions to the public good (Bergstrom et al. 1986). In this model, the usual equilibrium will exhibit free riding, meaning that there is a household member who abstains from contributing to the public good. In this case, the MWTP for the non-contributing member will be below the market price, while the MWTP for the contributing member will equal the market price. If, for a public good, both MWTP values are equal (and therefore equal to the market price), there will be a joint contribution to the particular public good. Interestingly, as shown by Cherchye et al. (2011b), this max condition can also be implemented using linear inequalities (containing integer variables). As such, the non-cooperative model is also verifiable using MILP methods.

The revealed preference model of household behavior has recently been incorporated into a broader marriage market model by Cherchye et al. (2017). The marriage market, which contains all potential partners, defines the 'outside options' of existing households. As these outside options give a lower bound on the utility each partner receives in his/her marriage, they will impact the intra-household allocation. The standard way to conceptualize the marriage market is as a two-sided matching model. In these models, the outside options are determined by the individual rationality and no blocking pairs conditions. Individual rationality imposes that no member of an existing household would prefer being single to staying married. The no blocking pairs condition requires that no currently unmarried couple would prefer to divorce their current partners and form new households. Cherchye et al. (ibid.) showed how these restrictions can be formalized as revealed preference restrictions on observed matching and consumption patterns. As an example, take a man $A$ and woman $B$ who are both married but not to each other. Let $A$ consume the bundle $\left(q_{A}, Q_{A}\right)$ in his current marriage, and let $B$ consume $\left(q_{B}, Q_{B}\right)$. Assume that if $A$ and $B$ were to form a couple, they would generate total income $Y_{A, B}$. Then, one revealed preference restriction that follows from this is:

$$
Y_{A, B} \leq p\left(q_{A}+q_{B}\right)+P \max \left\{Q_{A}, Q_{B}\right\}
$$

where again max is the element-wise maximum operator. To see that this restriction must hold, assume for a moment that the inequality is reversed, i.e. $Y_{A, B}>p\left(q_{A}+\right.$ $\left.q_{B}\right)+P \max \left\{Q_{A}, Q_{B}\right\}$. In this case, the 'new couple' $(A, B)$ could buy the bundle $\left(q_{A}, \max \left\{Q_{A}, Q_{B}\right\}\right)$ for $A$ and $\left(q_{B}, \max \left\{Q_{A}, Q_{B}\right\}\right)$ for $B$ and still have some money left to buy additional goods. In other words, by forming the couple $(A, B)$, both $A$ and $B$ can improve upon their current consumption bundle, which means that they form a blocking pair. $^{12}$

12 Of course, this disregards the fact that there are many other things in addition to the consumption of goods that influence the utility that partners derive from marriage. 


\subsection{Other Recent Contributions}

In this subsection, we provide a brief (non-exhaustive) list of other contributions to the revealed preference literature.

Functional Form Restrictions: Matzkin and Richter (1991) show that SARP is necessary and sufficient for the existence of a monotone, continuous and strictly concave utility function that rationalizes a data set. Chiappori and Rochet (1987) add differentiability to the rationalizing utility function and provide a necessary and sufficient condition for this case, which they call strong-SARP (SSARP). Varian (1983a) considers homothetic and additively separable utility functions. The first leads to the well-known homothetic axiom of revealed preference (HARP). Brown and Calsamiglia (2007) obtained revealed preference conditions for quasi-linear utility functions. This result is extended by Cherchye et al. (2015b), who examined the revealed preference conditions that are consistent with the generalized quasi-linear utility specification.

Uncertainty: Varian (1983b, 1988) and Green and Osband (1991) derived revealed preference conditions for the expected utility model. These were generalized to other models of decision making under uncertainty by Echenique and Saito (2015), Heufer (2014) and Polisson et al. (2017).

Intertemporal: Browning (1989) derived the revealed preference conditions for the standard life-cycle intertemporal consumption model. These were generalized to include models of habit formation and rational addiction by Crawford (2010) and Demuynck and Verriest (2013). Adams et al. (2014) derived the revealed preference conditions for the collective intertemporal model.

Behavioral Conditions: Chambers et al. (2010) examined revealed preference conditions for complementarity in choice behavior. Chambers et al. (2011) derived revealed preference conditions for substitutability, and Cherchye et al. (2018) obtained the revealed preference conditions under the assumption that goods are normal.

Stochastic Revealed Preference: The theory of stochastic revealed preferences was developed by McFadden and Richter (1971) (see McFadden 2005 for an overview). Stochastic revealed preference theory departs from the random utility model in which at each decision problem, an individual draws a utility function at random and chooses the best option according to this utility function. However, random utility models also have a population interpretation: there are many people with a fixed utility function, but each observation is obtained using a random draw from the population. Recently, there has been considerable interest in applying these revealed preference models to consumption data. Blundell et al. (2003, 2008) use non-parametric Engel curve estimation with revealed preference restrictions to obtain tight bounds on welfare and demand counterfactuals. Hoderlein and Stoye (2014) propose tests for the weak axiom of stochastic revealed preference (WASRP), which is a stochastic revealed preference version of Samuelson's WARP condition. Kitamura and Stoye (2018) and Kawaguchi (2017) verify stochastic 
revealed preference conditions that also take into account transitivity. Cosaert and Demuynck (2018) show how the WASRP can be used to derive counterfactual welfare and demand analysis.

Other Models: Brown and Matzkin (1996), Brown and Shannon (2000) and Brown and Kannan (2008) derived revealed preference conditions for general equilibrium models. Cherchye et al. (2011c) and Carvajal and Song (2018) provided simpler testable conditions for these models by exploiting the Varian inequalities. Demuynck and Seel (2018) derived revealed preference conditions for models with limited consideration. Cherchye et al. (2013), Carvajal and Gonzáles (2014) and Chambers and Echenique (2014) examined the revealed preference conditions for (Nash) bargaining models. Polisson and Quah (2013), Forges and Iehlé (2013), Forges and Iehlé (2014) and Cosaert and Demuynck (2015) considered revealed preference characterizations of discrete choice models. Matzkin (1991), Forges and Minelli (2009), Cherchye et al. (2014) and Nishimura et al. (2017) analyzed models with nonlinear budgets. Finally, van Bruggen and Heufer (2017) showed that GARP is a valid test of the utility maximization hypothesis in lab environments.

\section{Evaluating Revealed Preference Tests}

The ultimate objective of a revealed preference application is, of course, its implementation on real-life (or experimental) data. This means that the results of these applications have to be analyzed and evaluated. Fortunately, there exists a large array of tools to do so. We divide this section into three parts. First, we provide a brief overview of various goodness-of-fit measures that are used to evaluate the fit of a single particular data set to the revealed preference conditions. Next, we provide a discussion of three useful concepts that can be used to evaluate the fit of a revealed preference test to a collection of data sets, i.e. pass rate, power and predictive success. Finally, we show how the revealed preference tests can be accommodated to take into account measurement error.

\subsection{Goodness-of-Fit Measures}

Revealed preference tests are 'sharp' tests: they only tell us whether or not observed choices are consistent with some underlying model. In practice, however, it may well be that the test is very close to satisfying the revealed preference restrictions. As noted by Varian (1990), for most purposes, nearly optimizing behavior is just as good as exactly optimizing behavior. This calls for a goodness-of-fit measure that tells us how close observed behavior is to actually passing the revealed preference test. In this section, we discuss several such measures.

Efficiency Indices: The most popular goodness-of-fit measure is called the critical cost efficiency index (CCEI) or the Afriat efficiency index (AEI) (Afriat 1972). The AEI measures the severity of GARP violations by calculating the minimal expenditure adjustment necessary to render the choices consistent with GARP. Before, we defined the revealed preference relation $R_{D}$ in the following way:

$$
x^{t} R_{D} x^{s} \leftrightarrow p^{t} x^{t} \geq p^{t} x^{s} .
$$


The AEI works by relaxing this condition to

$$
x^{t} R_{D}(e) x^{s} \leftrightarrow e p^{t} x^{t} \geq p^{t} x^{s} .
$$

where $e \in[0,1]$. When $e=1$, we obtain the usual revealed preference restriction, $R_{D}(1)=R_{D}$. The lower the value of $e$, the less stringent the revealed preference relation, i.e. for $e \leq e^{\prime}: R_{D}\left(e^{\prime}\right) \subseteq R_{D}(e)$. Similarly, we can define GARP using the relation $R_{D}(e)$ instead of $R_{D}$. This gives us a GARP test, conditional on some value of $e$, denoted by $\operatorname{GARP}(e)$. The AEI is the highest value of $e$ such that the data set passes $\operatorname{GARP}(e)$. In practice, the AEI is easily calculated using a binary search algorithm (Varian 1990).

Varian (ibid.) also proposes a generalization of the AEI (called the Varian efficiency vector $(\mathrm{VEV})$ ) by letting the adjustment differ between observations. For a vector $E=$ $\left(e^{1}, \ldots, e^{T}\right)$ of numbers in $[0,1]$ we define the relation

$$
x^{t} R_{D}(E) x^{s} \leftrightarrow e^{t} p^{t} x^{t} \geq p^{t} x^{s} .
$$

A data set satisfies GARP $(E)$ if GARP holds when it is defined over the relation $R_{D}(E)$. The VEV is defined as the vector $E$ closest to the unit vector in some norm subject to the data satisfying GARP $(E)$. Thus, in practice, the VEV may differ for different norms. By exploiting the equivalence between GARP and the Varian inequalities in Theorem 1, Heufer and Hjertstrand (2019) proposed a computationally simple method to calculate the VEV by minimizing the linear objective function (i.e. in the L1-norm) $\frac{1}{T} \sum_{t=1}^{T}\left(1-e^{t}\right)$ subject to the MILP formulation of the Varian inequalities:

There exist numbers $V^{t} \in[0,1]$ and $e^{t} \in[0,1]$ and $X^{t, s} \in\{0,1\}$ such that for all observations $t, s$ :

$$
\begin{aligned}
& V^{t}-V^{s}<X^{t, s} \\
& \left(X^{t, s}-1\right) \leq V^{t}-V^{s}, \\
& e^{t} p^{t} x^{t}-p^{t} x^{s}<X^{t, s} A^{t}, \\
& \left(X^{t, s}-1\right) A^{s} \leq p^{s} x^{t}-e^{s} p^{s} x^{s} .
\end{aligned}
$$

It is easy to see that the AEI is a special case of the VEV, in which all adjustments are equal, i.e. $e=e^{t}$ for all $t=1, \ldots, T$. The AEI and VEV are both measures of wasted expenditure due to inconsistency with GARP: if the consumer has an AEI (VEV) less than one, then he could have obtained the same level of utility by only spending a fraction 1-AEI (1-VEV) of expenditures. In this regard, Varian (1990) interprets the AEI/VEV as measures of goodness-of-fit of the utility maximization model.

Heufer and Hjertstrand (2019) propose analogous indices for homothetic utility maximization, which they call the homothetic efficiency index (HEI) and the homothetic efficiency vector (HEV). Like the AEI/VEV, these measures can be interpreted as the amount of wasted expenditure. They also introduce the misspecification index (MSI), which is the additional adjustment required to make data that have already been adjusted to fit the utility maximization model also fit the homothetic utility maximization model. The MSI is defined as:

$$
\mathrm{MSI}=\frac{\mathrm{AEI}-\mathrm{HEI}}{\mathrm{AEI}} .
$$


As with the AEI, the HEI can be easily calculated using a binary search algorithm. Heufer and Hjertstrand (2019) also propose a procedure to calculate a first-order approximation to the HEV by solving an LP problem.

The Houtman-Maks Index: Houtman and Maks (1985) proposed measuring the degree of inconsistency as the maximal subset of observations consistent with revealed preference. Like the VEV, the Houtman-Maks (HM)-index is defined as the vector $E$ closest to the unit vector conditional on the data satisfying $\operatorname{GARP}(E)$. However, while the vector $E$ takes on continuous values in the $\mathrm{VEV}$, it is restricted to be binary for the HM-index, i.e. $e^{t} \in\{0,1\}$ for all $t$. Heufer and Hjertstrand (2015) propose two methods to calculate the HM-index. One method is based on a simple modification of the combinatorial algorithm in Gross and Kaiser (1996) and is applicable when the data consist of two goods. The second method is analogous to calculating the VEV and applicable to any number of goods. In this method, the HM-index is the value that minimizes $\frac{1}{T} \sum_{t=1}^{T}\left(1-e^{t}\right)$ subject to the MILP restrictions derived from the Varian inequalities, with the additional restriction that the vector $E$ be binary.

The Money Pump: Echenique et al. (2011) proposed an index based on the idea of the money pump, called the money pump index (MPI). The basic idea is that if an individual violates GARP, then she has a revealed preference cycle. This means that she is open to exploitation. Assume that a consumer has a revealed preference cycle, i.e. a set of observations $1, \ldots, J$ such that, for all $j \leq J-1$,

$$
p^{j} q^{j} \geq p^{j} q^{j+1} \text { and } p^{J} q^{J}>p^{J} q^{1}
$$

Intransitivity means that the consumer would also prefer $q^{j+1}$ to $q^{j}$, so a trader can easily give $q^{j+1}$ to the consumer in return for $q^{j}$ and cash the surplus $p^{j}\left(q^{j}-q^{j+1}\right)$. The total amount this trader can extract from the cycle is given by $\sum_{j=1}^{J-1} p^{j}\left(q^{j}-q^{j+1}\right)+p^{J}\left(q^{1}-q^{J}\right)$. Echenique et al. (2011) suggests interpreting the total amount of money that can thus be generated (divided by the total income over the cycle) as a measure of failure of the GARP test. The MPI index is the average of this measure over all cycles. Computing the MPI index, however, is computationally very cumbersome, i.e. NP-hard (Smeulders et al. 2013). ${ }^{13}$

Minimum Cost Index: Dean and Martin (2016) suggest measuring violations of GARP by considering the minimum cost necessary to break all revealed preference cycles, where the cost of removing a revealed preference comparison $x^{t} R_{D} x^{s}$ is given by

$$
p^{t}\left(x^{t}-x^{s}\right)
$$

The idea of this index, called the minimum cost index (MCI), is that if a cycle can be broken by removing only low-cost comparisons, then this is less serious than a cycle that involves only high-cost comparisons. Although the MCI is computationally hard, Dean and Martin (ibid.) show how it can be computed using off-the-shelf algorithms.

13 Computing the maximum or minimum of the measure over all cycles can be done in a computationally efficient manner (i.e. in polynomial time). 


\subsection{Pass Rate, Power and Predictive Success}

Revealed preference tests are not traditional tests. The reason is that revealed preference conditions are essentially set predictors: for a specific behavioral model, the revealed preference test predicts that observed choice behavior will lie within a certain bounded region of the consumption outcome space, namely the collection of all datasets that satisfy the particular revealed preference test.

For a traditional revealed preference test, the outcome space, $\Omega$, contains the set of all possible consumption bundles $\left(q^{t}\right)_{t=1, \ldots, T}$ that exhaust the consumer's budget at every observation $t$. The revealed preference test under consideration, e.g. GARP, then effectively bounds a region within this outcome space, say $A$,

$$
A=\left\{\left(q^{t}\right)_{t=1, \ldots, T}:\left(p^{t}, q^{t}\right)_{t=1, \ldots, T} \text { satisfies } G A R P\right\}
$$

Now, assume that we observe a collection of data sets where each data set is considered as an observation in the set $\Omega$. We find that some data sets are consistent with GARP, i.e. lie within the set $A$, while some other data sets are not consistent with GARP and therefore lie outside the set $A$. Of course, the higher the proportion of data sets in $A$, the better the model is supported empirically. The pass rate quantifies this empirical support as the fraction of data sets that are situated in the set $A$, i.e. satisfy GARP.

When treating observed data sets as random observations, the observed pass rate $\hat{\pi}$ will not be equal to the population pass rate $\pi$, although its asymptotic distribution is well known:

$$
\sqrt{n}(\hat{\pi}-\pi) \sim^{a} N(0, \pi(1-\pi)),
$$

where $n$ denotes the number of observations. However, pass rates only capture one dimension of the empirical performance of a revealed preference test. In general, the pass rate of the model will be higher the larger the set $A$. Therefore, for a revealed preference test to be meaningful, we would like this set to be sufficiently small. The smallness of the set $A$ defines the discriminatory power of the GARP test. The power is commonly expressed as the relative size of the complement of $A$. This immediately allows for an intuitive probabilistic interpretation: the power measures the probability that a data set generated by irrational behavior (i.e. behavior that is inconsistent with the underlying model of choice) violates the revealed preference condition. To quantify this power, we therefore need a model of irrational behavior. The most common practice in revealed preference theory defines irrational behavior as random draws from the outcome space $\Omega$. This generates the power measure as defined by Bronars (1987). Bronars motivated his measure as an operationalization of Becker (1962)'s theoretical notion of irrational behavior that states that consumers randomly (uniformly) choose consumption bundles that exhaust their available budget. It has become standard practice in empirical applications of revealed preference to report the Bronars power measure, although other power measures are also available (see Andreoni et al. 2013).

In general, the pass rate and the power are two sides of the same coin. A favorable pass rate for a specific model provides convincing support only if the associated test has high enough power. In practice, the two measures are almost always inversely correlated, which makes it interesting to define a summarizing measure that combines the two into a single metric. Beatty and Crawford (2011) suggest such a measure based on an original 
idea of Selten (1991). This measure is called the predictive success and is defined as:

$$
\text { predictive success }=\text { pass rate }-(1-\text { power }) .
$$

Pass rates and power are always between 0 and 1, which means that the predictive success is bounded between -1 and 1 . A value close to 1 indicates a model that is consistent with a large fraction of the observations and also has high power. By contrast, a value close to -1 implies a model with very low power and low pass rate. In this case, the revealed preference model allows for almost all (random) behavior and yet the observed data fails to pass the test. Finally, a predictive success close to 0 corresponds to a model where the pass rate for the observed behavior is more or less equal to what the expected pass rate would be if behavior were random. Essentially, it implies that the rationality test does not allow us to distinguish observed behavior from random (irrational) behavior. In general, for a model to be meaningful, the predictive success should be above 0. Demuynck (2015) shows how to conduct statistical inference on predictive success measures to compare the empirical fit between different revealed preference models.

\subsection{Measurement Errors}

The presence of measurement errors in observed data may erroneously result in a violation of revealed preference even when the 'true' data (without errors) satisfy the revealed preference restrictions. Suppose that the observed quantity data $x^{t} \in \mathbb{R}^{N}$ is measured with error and let us denote the 'true' (unobserved) data by $q^{t} .{ }^{14}$ Following Varian (1985), we assume that the observed and 'true' data are related via the multiplicative Berkson measurement error model:

$$
q^{t}=x^{t}\left(1+\varepsilon^{t}\right)
$$

where the random measurement errors at observation $t$ are denoted by $\varepsilon^{t} \in \mathbb{R}^{N}$. We wish to test the following hypothesis:

$$
\mathrm{H}_{0}: \text { The data set }\left(p^{t}, q^{t}\right)_{t=1, \ldots, T} \text { satisfies GARP. }
$$

If the observed data $\left(p^{t}, x^{t}\right)_{t=1, \ldots, T}$ violate GARP but one fails to reject $\mathrm{H}_{0}$, then it means that failure of the GARP test might be attributed to measurement error. In contrast, if $\mathrm{H}_{0}$ is rejected, then it means that the violation of GARP is too strong to be attributed to measurement error alone.

Varian (ibid.) departs from the assumption that $\varepsilon_{n}^{t} \sim N\left(0, \sigma^{2}\right)$ are i.i.d. ${ }^{15}$ Then, under $H_{0}$ and this distributional assumption, we have that $\left(p^{t}, x^{t}\left(1+\varepsilon^{t}\right)\right)_{t=1, \ldots, n}$ satisfies GARP and the statistic,

$$
M=\sum_{t=1}^{T} \sum_{n=1}^{N} \frac{\varepsilon_{n}^{t} \varepsilon_{n}^{t}}{\sigma^{2}},
$$

\footnotetext{
${ }^{14}$ It is straightforward to modify the exposition and instead allow for measurement error in prices (see, for example, Jones and Edgerton 2009).

${ }^{15}$ We use superscripts to refer to observations, while subscripts refer to the goods.
} 
has a $\chi^{2}$ distribution with $T N$ degrees of freedom. Let $c_{\alpha}$ be the $(1-\alpha) \times 100$ th percentile of this distribution. If measurement errors were observed, we would reject $H_{0}$ if

$$
M>c_{\alpha}
$$

Unfortunately, we do not observe the measurement errors, $\varepsilon_{n}^{t}$, which means that we cannot compute $M$. Instead, Varian proposed looking for the errors $e_{n}^{t}$ that solve

$$
\widehat{M}=\min \sum_{t=1}^{T} \sum_{n=1}^{N} e_{n}^{t} e_{n}^{t}, \text { s.t. }\left(p^{t}, x^{t}\left(1+e^{t}\right)\right)_{t=1, \ldots, T} \text { satisfies GARP. }
$$

$\widehat{M}$ gives the minimal distance (in a squared Euclidean sense) from the observed dataset to the collection of data sets that satisfy GARP. Under $\mathrm{H}_{0}$, the true data satisfy GARP, which means that:

$$
\widehat{M} \leq \sum_{t=1}^{T} \varepsilon^{t} \varepsilon^{t}
$$

This shows that we can form a conservative test that rejects $H_{0}$ whenever

$$
\frac{\widehat{M}}{\sigma^{2}}>c_{\alpha}
$$

This test still hinges on the condition that the error variance $\sigma^{2}$ is known. Of course, in empirical applications, this is never the case. Varian therefore proposed an alternative 'Bayesian'-style approach. Notice that the rejection criterion can be restated as

$$
\sigma^{2}<\frac{\widehat{M}}{c_{\alpha}}
$$

Varian defined the statistic $\overline{\sigma^{2}}=\frac{\widehat{M}}{c_{\alpha}}$, which provides the largest value of $\sigma^{2}$ for which $\mathrm{H}_{0}$ would be rejected. In other words, if $\overline{\sigma^{2}}$ is smaller than your prior belief concerning the variance $\sigma^{2}$, you may want to accept the hypothesis that the 'true' data $\left(p^{t}, q^{t}\right)_{t=1, \ldots, T}$ satisfy GARP.

A main difficulty in implementing the test involves solving (7). Varian (1985)'s implementation is based on the Afriat inequalities, which would require solving a highly non-linear optimization problem. However, by exploiting the equivalence between GARP and the Varian inequalities in Theorem 1, we obtain a considerably simpler problem, since the variables $e_{n}^{t}$ enter the Varian inequalities linearly. This means that we can use the MILP formulation of the Varian inequalities when solving problem (7). This amounts to minimizing a quadratic (and convex) objective function subject to a set of linear constraints, which can be done using off-the-shelf algorithms.

The Upper Bound Test: Fleissig and Whitney (2005) proposed an alternative procedure to test hypothesis (6) that was further modified by Jones and Edgerton (2009). The test statistic of Jones and Edgerton's procedure is defined as the smallest value of $F$ such that the following adjustments of the Afriat inequalities hold:

$$
U^{s}-U^{t}-\lambda^{t} p^{t}\left(x^{s}-x^{t}\right) \leq \lambda^{t} F .
$$


Assuming that the observed and 'true' data are related via the multiplicative Berkson measurement error model (5), Jones and Edgerton show that under $\mathrm{H}_{0}$ :

$$
F \leq C \equiv \max _{s, t=1, \ldots, T}\left\{p^{t}\left(x^{s} \circ \varepsilon^{s}-x^{t} \circ \varepsilon^{t}\right)\right\}
$$

where $\circ$ denotes the Hadamard (elementwise) product. For a given distribution of the measurement errors, it is straightforward to simulate the distribution of $C$ and obtain its $(1-\alpha) \times 100$ th percentile $c_{\alpha}^{\prime}$. The null of rationality, $\mathrm{H}_{0}$, is then rejected whenever $F$ exceeds this critical value, i.e. $F>c_{\alpha}^{\prime}$. Compared to the MILP procedure above, the upper bound procedure is much easier to compute. However, the test is more conservative than Varian's.

Hjertstrand (2013) pointed out that the test statistic $F$ can be calculated by solving a sequence of linear programming problems in the form of a binary search. He also noted that this is the most computationally expensive part of the implementation. By exploiting the equivalence between the Afriat inequalities and GARP in Theorem 1, Hjertstrand showed that $F$ can be calculated using a combinatorial algorithm very similar to GARP. This allows for a very efficient implementation of the procedure and, consequently, its applicability to large data sets.

Finally, Hjertstrand (ibid.) noted that the Berkson measurement error model in (5), where the 'true' variable of interest is predicted (or caused) by the observed variable and a random error, may be inappropriate to describe many economic data sets. Instead, the classical measurement error model, where the observed variable is predicted by the 'true' variable and a random error, i.e.

$$
x^{t}=q^{t}\left(1+\epsilon^{t}\right)
$$

is widely considered a more appropriate model in economic applications. Hjertstrand showed how the upper bound test can be modified to test $\mathrm{H}_{0}$ under the assumption of this classical multiplicative measurement error model.

\section{Conclusion}

Revealed preference theory has expanded considerably since Samuelson's seminal contribution more than 80 years ago. Nowadays, revealed preference theory has grown into a very versatile theoretical and empirical toolbox that can be used to study a wide variety of decision making models.

In this chapter we have provided a brief survey of several recent contributions to the literature with a special emphasis on computational advancements. In particular, we have shown that many revealed preference models can efficiently be implemented using mixed integer linear programming procedures. This allows us to bring various popular decision making models, that were previously thought as being unverifiable, to the data. As a result, this not only adds to the attractiveness of revealed preference theory as an empirical tool, but it also allows us to expand the revealed preference toolbox to new and unexplored models in economics. The next 80 years look promising indeed. 


\section{References}

Adams, A., L. Cherchye, B. De Rock and E. Verriest (2014) "Now or Later? Time Inconsistency, Collective Choice and Revealed Preference," American Economic Review, 104: $4,147-4,183$.

Afriat, S.N. (1967) "The Construction of Utility Functions From Expenditure Data," International Economic Review, 8: 67-77.

Afriat, S.N. (1969) "The Construction of Separable Utility Functions From Expenditure Data," Tech. rep., University of North Carolina.

Afriat, S.N. (1972) "Efficiency Estimation of Production Functions," International Economic Review, 13: 568-598.

Andreoni, J., B.J. Gillen and W.T Harbaugh (2013) "The Power of Revealed Preference Tests: Ex-Post Evaluation of Experimental Design," Tech. rep., University of California, San Diego.

Apps, P.F. and R. Rees (1988) "Taxation and the Household," Journal of Public Economics, 35: 355-369.

Beatty, T.K.M. and I. Crawford (2011) "How Demanding is the Revealed Preference Approach to Demand," American Economic Review, 101: 2,782-2,795.

Becker, G.S. (1962) "Irrational Behavior and Economic Theory," Journal of Political Economy, 70: 1-13.

Belongia, M.T. and K.A. Chrystal (1991) "An Admissible Monetary Aggregate for the United Kingdom," Review of Economics and Statistics, 73: 497-503.

Bergstrom, T.C., L. Blume and H.R. Varian (1986) "On the Private Provision of Public Goods," Journal of Public Economics, 29: 25-49.

Blundell, R., M. Browning and I. Crawford (2003) "Nonparametric Engel Curves and Revealed Preference," Econometrica, 71: 205-240.

Blundell, R., M. Browning and I. Crawford (2008) "Best Nonparametric Bounds on Demand Responses," Econometrica, 76: 1,227-1,262.

Bronars, S.G. (1987) "The Power of Nonparametric Tests of Preference Maximization," Econometrica, 55: 693-698.

Brown, D.J. and C. Calsamiglia (2007) "The Nonparametric Approach to Applied Welfare Analysis," Economic Theory, 31: 183-188.

Brown, D. and R. Kannan (2008) "Two Algorithms for Solving the Walrasian Equilibrium Inequalities," in D. Brown and F. Kubler (eds) Computational Aspects of General Equilibrium Theory: Refutable Theories of Value. Lecture Notes in Economics and Mathematical Systems. Berlin, Springer-Verlag. 
Brown, D.J. and R.L. Matzkin (1996) "Testable Restrictions on the Equilibrium Manifold," Econometrica, 64: 1,249-1,262.

Brown, D.J. and C. Shannon (2000) "Uniqueness, Stability, and Comparative Statics in Rationalizable Walrasian Markets," Econometrica, 68: 1,529-1,540.

Browning, M. (1989) "A Nonparametric Test of the Life-Cycle Rational Expectations Hypothesis," International Economic Review, 30: 979-992.

van Bruggen, P. and J. Heufer (2017) "Afriat in the Lab," Journal of Economic Theory, 169: 546-550.

Carvajal, A. and N. Gonzáles (2014) "On Refutability of the Nash Bargaining Solution," Journal of Mathematical Economics, 50: 177-186.

Carvajal, A. and X. Song (2018) "Testing Pareto Efficiency and Competitive Equilibrium in Economies with Public Goods," Journal of Mathematical Economics, 75: 19-30.

Chambers, C.P. and F. Echenique (2014) "On the Consistency of Data With Bargaining Theories," Theoretical Economics, 9: 137-162.

Chambers, C.P. and F. Echenique (2016) Revealed Preference Theory. Cambridge, England, Cambridge University Press.

Chambers, C.P., F. Echenique and E. Shmaya (2010) "On Behavioural Complementarities and its Implications," Journal of Economic Theory, 145: 2,332-2,355.

Chambers, C.P., F. Echenique and E. Shmaya (2011) "Testable Implications of Gross Substitutes in Demand for Two Goods," American Economic Journal: Microeconomics, 3 : 129-136.

Cherchye, L., I. Crawford, B. De Rock and F. Vermeulen (2009a) "The Revealed Preference Approach to Demand," in D. Slottje (ed.) Quantifying Consumer Preferences: Estimating Demand Systems. Contributions to Economic Analysis. Bingley, England, Emerald Press.

Cherchye, L., B. De Rock, T. Demuynck and F. Vermeulen (2017) "Household Consumption when the Marriage is Stable," American Economic Review, 107: 1,507-1,534.

Cherchye, L., B. De Rock and F. Vermeulen (2007) "The Collective Model of Household Consumption: A Nonparametric Characterization," Econometrica, 75: 553-574.

Cherchye, L., B. De Rock and F. Vermeulen (2009b) "Opening the Black box of IntraHousehold Decision-Making: Theory and Nonparametric Empirical Tests of General Collective Consumption Models," Journal of Political Economy, 117: 1,074-1,104.

Cherchye, L., B. De Rock and F. Vermeulen (2011a) "The Revealed Preference Approach to Collective Consumption Behavior: Testing and Sharing Rule Recovery," Review of Economic Studies, 78: 176-198.

Cherchye, L., T. Demuynck and B. De Rock (2011b) "Revealed Preference Analysis of Noncooperative Household Consumption," Economic Journal, 121: 1,073-1,096. 
Cherchye, L., T. Demuynck and B. De Rock (2011c) "Testable Implications of General Equilibrium Models: An Integer Programming Approach," Journal of Mathematical Economics, 47: 564-575.

Cherchye, L., T. Demuynck and B. De Rock (2013) "Nash Bargained Consumption Decisions: A Revealed Preference Analysis," Economic Journal, 123: 195-235.

Cherchye, L., T. Demuynck and B. De Rock (2014) "Revealed Preference Analysis for Convex Rationalization on Nonlinear Budget Sets," Journal of Economic Theory, 152: 224-236.

Cherchye, L., T. Demuynck, B. De Rock and P. Hjertstrand (2015a) "Revealed Preference Tests for Weak Separability: An Integer Programming Approach," Journal of Econometrics, 186: 129-141.

Cherchye, L., T. Demuynck and B. De Rock (2015b) "Is Utility Transferable? A Revealed Preference Analysis," Theoretical Economics, 10: 51-65.

Cherchye, L., T. Demuynck and B. De Rock (2018) "Normality of Demand in a Twogoods Setting," Journal of Economic Theory, 173: 361-382.

Chiappori, P.-A. (1988) "Rational Household Labor Supply," Econometrica, 56: 63-89.

Chiappori, P.-A. (1992) "Collective Labor Supply and Welfare," Journal of Political Economy, 100: 437-467.

Chiappori, P.-A. and J.-C. Rochet (1987) "Revealed Preferences and Differentiable Demand," Econometrica, 55: 687-691.

Cosaert, S. and T. Demuynck (2015) "Revealed Preference Theory for Finite Choice Sets," Economic Theory, 59: 169-200.

Cosaert, S. and T. Demuynck (2018) "Nonparametric Welfare and Demand Analysis with Unobserved Individual Heterogeneity," Review of Economic and Statistics, 100: 349-361.

Crawford, I. (2010) "Habits Revealed," Review of Economic Studies, 77: 1,382-1,402.

Crawford, I. and B. De Rock (2014) "Empirical Revealed Preference," Annual Review of Economics, 6: 503-524.

Dean, M. and D. Martin (2016) "Measuring Rationality with the Minimum Cost of Revealed Preference Violations," Review of Economics and Statistics, 98: 524-534.

Demuynck, T. (2015) "Statistical Inference for Measures of Predictive Success," Theory and Decision, 79: 689-699.

Demuynck, T. and C. Seel (2018) "Revealed Preference With Limited Consideration," American Economic Journal: Microeconomics, 10: 102-131. 
Demuynck, T. and E. Verriest (2013) "I'll Never Forget my First Cigarette: A Revealed Preference Analysis of the Habits as Durables Model," International Economic Review, 54: 717-738.

Diewert, W.E. (1973) "Afriat and Revealed Preference Theory," Review of Economic Studies, 40: 419-425.

Diewert, W.E. (2012) "Afriat's Theorem and Some Extensions to Choice Under Uncertainty," Economic Journal, 122: 305-331.

Diewert, W.E. and C. Parkan (1985) "Tests for the Consistency of Consumer Data," Journal of Econometrics, 30: 127-147.

Echenique, F. (2014) "Testing for Separability is Hard," Tech. rep., Caltech.

Echenique, F., S. Lee and M. Shum (2011) "The Money Pump as a Measure of Revealed Preference Violations," Journal of Political Economy, 119: 1,201-1,223.

Echenique, F. and K. Saito (2015) "Savage in the Market," Econometrica, 83: 1,467-1,495.

Elger, C.T., B.E. Jones, D.L. Edgerton and J.M. Binner (2008) "A Note on the Optimal Level of Monetary Aggregation in the United Kingdom," Macroeconomic Dynamics, 12: $117-131$.

Fisher, D. and A.R. Fleissig (1997) "Monetary Aggregation and the Demand for Assets," Journal of Money, Credit and Banking, 29: 458-475.

Fleissig, A.R. and G.A. Whitney (2003) "A New PC-Based Test for Varian's Weak Separability Condition," Journal of Business and Economic Statistics, 21: 133-145.

Fleissig, A.R. and G.A. Whitney (2005) "Testing for the Significance of Violations of Afriat's Inequalities," Journal of Business and Economic Statistics, 23: 355-362.

Forges, F. and V. Iehlé (2013) "Essential Data, Budget Sets and Rationalization," Economic Theory, 54: 449-461.

Forges, F. and V. Iehlé (2014) "Afriat's Theorem for Indivisible Goods," Journal of Mathematical Economics, 54: 1-6.

Forges, F. and E. Minelli (2009) "Afriat's Theorem for General Budget Sets," Journal of Economic Theory, 144: 135-145.

Ginsberg, W. (1973) "Concavity and Quasiconcavity in Economics," Journal of Economic Theory, 6: 596-605.

Green, E.J. and K. Osband (1991) "A Revealed Preference Theory for Expected Utility," Review of Economic Studies, 58: 677-695.

Gross, J. and D. Kaiser (1996) "Two Simple Algorithms for Generating a Subset of Data Consistent with WARP and Other Binary Relations," Journal of Business and Economic Statistics, 14: 251-255. 
Heufer, J. (2014) "Nonparametric Comparative Revealed Risk Aversion," Journal of Economic Theory, 153: 569-616.

Heufer, J. and P. Hjertstrand (2015) "Consistent Subsets: Computationally Feasible Methods to Compute the Houtman-Maks-Index," Economics Letters, 128: 87-89.

Heufer, J. and P. Hjertstrand (2019) "Homothetic Efficiency: Theory and Applications," forthcoming in Journal of Business and Economic Statistics.

Hjertstrand, P. (2009) "A Monte Carlo Study of the Necessary and Sufficient Conditions for Weak Separability," Advances in Econometrics, 24: 151-182.

Hjertstrand, P. (2013) "A Simple Method to Account for Measurement Errors in Revealed Preference Tests," IFN Working Paper 990, Research Institute of Industrial Economics, Stockholm.

Hjertstrand, P. and J.L. Swofford (2012) "Revealed Preference Tests for Consistency with Weakly Separable Indirect Utility," Theory and Decision, 72: 245-256.

Hjertstrand, P. and J.L. Swofford (2019) "Revealed Preference Tests of Indirect and Homothetic Weak Separability of Financial Assets, Consumption and Leisure," forthcoming in Journal of Financial Stability.

Hjertstrand, P., J.L. Swofford and G.A. Whitney (2016) "Mixed Integer Programming Revealed Preference Tests of Utility Maximization and Weak Separability of Consumption, Leisure and Money," Journal of Money, Credit and Banking, 48: 1,547-1,561.

Hjertstrand, P., J.L. Swofford and G.A. Whitney (2019) "Index Numbers and Revealed Preference Rankings," forthcoming in Macroeconomic Dynamics.

Hoderlein, S. and J. Stoye (2014) "Revealed Preference in a Heterogeneous Population," Review of Economics and Statistics, 96: 197-213.

Houthakker, H.S. (1950) "Revealed Preference and the Utility Function," Economica, 17: 159-174.

Houtman, M. and J.A.H. Maks (1985) "Determining all Maximal Data Subsets Consistent with Revealed Preference," Kwantitatieve Methoden, 89: 89-104.

Jones, B.E., D. Dutkowsky and C.T. Elger (2005) "Sweep Programs and Optimal Money Aggregation," Journal of Banking and Finance, 29: 483-508.

Jones, B.E. and D.L. Edgerton (2009) "Testing Utility Maximization With Measurement Errors in the Data," Advances in Econometrics, 24: 199-236.

Kawaguchi, K. (2017) "Testing Rationality Without Restricting Heterogeneity," Journal of Econometrics, 197: 153-171.

Kitamura, Y. and J. Stoye (2018) "Nonparametric Analysis of Random Utility Models," Econometrica, 86: 1,883-1,909. 
Matzkin, R.L. (1991) "Axioms of Revealed Preference for Nonlinear Choice Sets," Econometrica, 59: 1,779-1,786.

Matzkin, R.L. and M.K. Richter (1991) "Testing Strictly Concave Rationality," Journal of Economic Theory, 53: 287-303.

McFadden, D.L. and M.K. Richter (1971) "On the Extension of a Set Function on a Set of Events to a Probability on the Generated Boolean $\sigma$-Algebra," Tech. rep., University of California, Berkeley.

McFadden, D.L. (2005) "Revealed Stochastic Preference: A Synthesis," Economic Theory, 26: 245-264.

Nishimura, H., E.A. Ok and J.K.H. Quah (2017) "A Comprehensive Approach to Revealed Preference Theory," American Economic Review, 107: 1,239-1,263.

Polisson, M. and J.K.H. Quah (2013) "Revealed Preference in a Discrete Consumption Space," American Economic Journal: Microeconomics, 5: 28-34.

Polisson, M., J.K.H. Quah and L. Renou (2017) "Revealed Preferences Over Risk and Uncertainty," School of Economics and Finance Discussion Paper 1706, University of St Andrews.

Samuelson, P.A. (1938) "A Note on the Pure Theory of Consumer's Behavior," Economica, 5: 61-71.

Samuelson, P.A. (1948) "Consumption Theory in Terms of Revealed Preference," Economica, 15: 243-253.

Selten, R. (1991) "Properties of a Measure of Predictive Success," Mathematical Social Sciences, 21: 153-167.

Smeulders, B., L. Cherchye, B. De Rock and F.C.R. Spieksma (2013) "The Money Pump as a Measure of Revealed Preference Violations: A Comment," Journal of Political Economy, 121: 1,248-1,258.

Swofford, J.L. and G.A. Whitney (1987) "Nonparametric Tests of Utility Maximization and Weak Separability for Consumption, Leisure and Money," Review of Economics and Statistics, 69: 458-464.

Swofford, J.L. and G.A. Whitney (1994) "A Revealed Preference Test for Weakly Separable Utility Maximization With Incomplete Adjustment," Journal of Econometrics, 60: 235-249.

Varian, H.R. (1982) "The Nonparametric Approach to Demand Analysis," Econometrica, 50: $945-974$.

Varian, H.R. (1983a) "Non-Parametric Tests of Consumer Behavior," Review of Economic Studies, 50: 99-110.

Varian, H.R. (1983b) "Nonparametric Tests of Models of Investor Behavior," Journal of Financial and Quantitative Analysis, 18: 269-278. 
Varian, H.R. (1984) "The Nonparametric Approach to Production Analysis," Econometrica, 52: 579-597.

Varian, H.R. (1985) "Non-Parametric Analysis of Optimizing Behaviour With Measurement Error," Journal of Econometrics, 30: 445-458.

Varian, H.R. (1988) "Estimating Risk Aversion From Arrow-Debreu Portfolio Choice," Econometrica, 56: 973-979.

Varian, H.R. (1990) "Goodness-of-Fit in Optimizing Models," Journal of Econometrics, 46: $125-140$.

Varian, H.R. (2006) "Revealed Preference," in M. Szenberg, L. Ramrattan and A.A Gottesman (eds.) Samuelson Economics and the Twenty-First Century. Oxford, Oxford University Press.

Warshall, S. (1962) "A Theorem of Boolean Matrices," Journal of the American Association of Computing Machinery, 9: 11-12. 